Factibilidad del uso de contratos de futuros del Chicago Mercantile Exchange para la cobertura del riesgo de precio en el ganado bovino chileno

Ricardo Troncoso-Sepúlveda y Juan Cabas-Monje 
Lecturas de Economía, 90 (enero-junio 2019), pp. 9-44

Ricardo Troncoso-Sepúlveda y Juan Cabas-Monje

Factibilidad del uso de contratos de futuros del Chicago Mercantile Exchange para la cobertura del riesgo de precio en el ganado bovino chileno

Resumen: El objetivo de este artículo es estudiar la factibilidad del uso de contratos de futuros del Chicago Mercantile Exchange como instrumento de cobertura del riesgo de precio para el ganado bovino en Chile. Para tal propósito, se realizaron pruebas de raíces unitarias estacionales y se utilizó el modelo Johnson-Stein para estimar las ratios de cobertura de mínimo riesgo entre 1975 y 2012. Los resultados muestran que los mercados de ganado bovino están integrados y que la ratio de cobertura óptima para un productor de ganado se encuentra en linea con las ratios estimadas para otros commodities. Estos hallazgos pueden ser útiles para hacedores de política agrícola en países en desarrollo porque confirman el potencial de este tipo de instrumentos para reducir el riesgo de precio entre los productores ganaderos y dan argumentos empíricos para fomentar su utilización.

Palabras clave: precios de futuros, mercados financieros internacionales, criterios para la toma de decisiones bajo riesgo e incertidumbre, política agrícola.

Clasificación JEL: G13, G15, D81, Q18.

Feasibility of using futures contracts of the Chicago Mercantile Exchange for hedging price risk in Chilean cattle

Abstract: The aim of this paper is to study the feasibility of using Chicago Mercantile Exchange futures contracts as a price risk hedging instrument for cattle in Chile. For this purpose, seasonal unit root tests were performed, and the Johnson-Stein model was used to estimate the minimum risk hedge ratios between 1975 and 2012. The results show that the cattle markets are integrated and that the optimal hedge ratio for a livestock, producer is in line with the estimated ratios for other commodities. These findings can be useful for agricultural policy makers in developing countries because they confirm the potential of this type of instrument to reduce the price risk. for livestock producers and provide empirical arguments to encourage its use.

Keywords: futures pricing, international financial markets, criteria for decision-making under risk and uncertainty, agricultural policy.

JEL Classification: G13, G15 D81, Q18.

Possibilité d'utiliser des contrats à terme sur Chicago Mercantile Exchange pour couvrir le risque de prix des bovins chiliens

Résumé: L'objectif de cet article est d'étudier la possibilité d'utiliser les contrats à terme de la Chicago Mercantile Exchange comme instrument de couverture du risque de prix des bovins au Chili. À cette fin, des tests de racine unitaire saisonniers ont été effectués et le modèle de Johnson-Stein a été utilisé pour estimer les ratios de couverture de risque minimum entre les années 1975 et 2012. Les résultats montrent que les marchés du bétail sont intégrés et que le ratio de couverture optimal pour un éleveur correspond aux ratios estimés pour les autres commodities. Ces résultats peuvent être utiles aux décideurs politiques agricoles des pays en développement, car ils confirment le potentiel de ce type d'instruments pour réduire le risque de prix des producteurs de bétail et fournissent des arguments empiriques pour encourager leur utilisation.

Mots clés: prix à terme, marchés financiers internationaux, critères de décision en cas de risque et d'incertitude, politique agricole.

Classification JEL: G13, G15, D81, Q18. 


\title{
Factibilidad del uso de contratos de futuros del Chicago Mercantile Exchange para la cobertura del riesgo de precio en el ganado bovino chileno*
}

\author{
Ricardo Troncoso-Sepúlveda (iD ${ }^{\mathrm{a}}$ y Juan Cabas-Monje ${ }^{\mathrm{b}}$
}

-Introducción. -I. El mercado de fututos en Chile. -II. Modelos de cobertura óptima con futuros. -III. Estimación de cobertura óptima con futuros. -Conclusiones. -Referencias.

doi: 10.17533/udea.le.n90a01

Primera versión recibida el 4 de mayo de 2018; versión final aprobada el 30 de julio de 2018

\section{Introducción}

La actividad ganadera está expuesta a múltiples riesgos que amenazan los ingresos de los productores, dificultan la planificación a mediano y largo plazo, y afectan los costos y la productividad de los planteles. Una variable importante al momento de tomar decisiones productivas es el precio y, en particular, su volatilidad, la cual ha sido un problema característico en Chile, que, si bien se ha reducido gracias a las condiciones favorables de la industria internacional, aún persiste y hace necesaria la búsqueda de mecanismos que permitan reducir el riesgo y ayuden a los productores a concentrarse en mejorar los rendimientos de sus planteles. Entre estos mecanismos se encuentran

* Este artículo es producto del proyecto de investigación del mismo nombre presentado por el primer autor para optar al grado de Magíster en Agronegocios, Universidad del Bío-Bío, Chillán, Chile.

a Ricardo Troncoso-Sepúlveda: doctorando en Economía y asistente de investigación, Universidad del Rosario, Bogotá, Colombia. Dirección postal: Calle 12C N 6-25, CP: 111711-111711201, Bogotá D.C. Colombia. Dirección electrónica: ricardo.troncoso@urosario.edu.co. https://orcid.org/0000-0002-4573-8231

b Juan Cabas-Monje: director del Centro de Investigación y Desarrollo en Agronegocios, Departamento de Gestión Empresarial, Facultad de Ciencias Empresariales, Universidad del Bío-Bío, Chillán, Chile. Dirección postal: Avda. Andrés Bello 720, Casilla 447-CP: 3800708, Chillán, Chile. Dirección electrónica: jcabas@ubiobio.cl. 
Troncoso y Cabas: Factibilidad del uso de contratos de futuros del Chicago Mercantile...

los derivados financieros, los cuales, como su nombre lo indica, son productos cuyo valor deriva de otro instrumento o activo, que puede ser un índice bursátil, acciones de una empresa o un commodity.

La utilización de derivados puede significar una reducción importante del riesgo de precio para las empresas del rubro agropecuario (Manzana, 2009). Así mismo, diversos autores han estudiado la efectividad del uso de derivados financieros como instrumentos de cobertura: Rolfo (1980) estimó estrategias de cobertura con futuros para productores representativos de cacao de Ghana, Nigeria, Costa de Marfil y Brasil usando contratos de la Bolsa de Londres. Los resultados mostraron que la ratio de cobertura óptima debe ser muy inferior a la unidad, y que la definición tradicional de cobertura que recomienda lo contrario necesita ser corregida, sobre todo en el contexto de productores que enfrentan incertidumbre en precio y cantidad. Wolf (1987) analizó portafolios óptimos de cobertura con contratos futuros y opciones sobre commodities, y usó estática comparativa para examinar los efectos de cambios en algunas variables sobre las posiciones óptimas, concluyendo que las opciones sobre commodities pueden ser utilizadas por agentes aversos al riesgo para reducir la incertidumbre. Noussinov y Leuthold (1999) comprobaron si la teoría de cobertura multiproducto genera valor para los alimentadores de ganado de Estados Unidos, y si es factible de ser implementada para reducir el riesgo de precio. Los autores concluyen que todas las estrategias de cobertura reducen el riesgo, pero que la cobertura multiproducto puede dar lugar a mejoras sustanciales. Arias y Segura (2000) estudiaron la factibilidad del uso de los mercados de futuros como instrumentos de cobertura de variación de precios de las bolsas de físicos. Utilizaron precios de maíz y trigo de la Bolsa de Cereales de Buenos Aires y del mercado mayorista de Chile, y determinaron relaciones cuantitativas y factibilidad técnica de cobertura en el mercado argentino de futuros. Zurita (2002) exploró diferentes razones de cobertura de mínima varianza entre contratos a plazo y futuros para distintos tipos de activos subyascentes. Da Silva, Aguiar y De Lima (2003) investigaron la efectividad de la cobertura de aceite de soya, harina de soya y soya brasileña en el Chicago Board of Trade (CBOT) y en la Bolsa Brasileña de Materias Primas y Futuros (BM\&F) para reducir el riesgo de pérdidas financieras provocadas por fluctuaciones de precios de las materias primas. Mostraron que 
una estrategia de cobertura cruzada mediante contratos futuros CBOT genera resultados más favorables para las operaciones de cobertura de aceite y harina de soya en relación con los futuros BM\&F. Milanesi, Miliozzi y Esandi (2010) analizaron el concepto de administración del riesgo y el efecto que este tiene en la rentabilidad de las empresas, y expusieron desde un punto de vista teórico la ratio de cobertura y su determinación. La Dirección de Investigaciones Científicas y Tecnológicas de la Universidad Católica de Chile (DICTUC, 2011) realizó un estudio en el que evaluó estrategias alternativas de cobertura ante fluctuaciones del precio del trigo en Chile. A través del cálculo de un coeficiente de efectividad de la cobertura, determinaron que la estrategia con futuros es la que permite reducir más el riesgo, asegurando también un ingreso mínimo más alto, pero su aplicabilidad es más compleja dados los altos requerimientos de liquidez para cubrir los llamados de margen debido a las fluctuaciones en la Chicago Mercantile Exchange. Conlon, Cotter y Gençay (2016) examinaron el impacto de las preferencias sobre las estrategias de cobertura óptima y el rendimiento para contratos futuros de café, algodón, maíz y petróleo crudo en bolsas de derivados de Estados Unidos. Sus resultados revelaron variaciones sustanciales en la ratio de cobertura entre distintos niveles de preferencias hacia el riesgo. En particular, individuos con alta aversión al riesgo tienden a reducir el portafolio de cobertura, pero logran una utilidad inferior en comparación con aquellos con baja aversión.

El propósito de este trabajo es determinar la factibilidad de uso de futuros del Chicago Mercantile Exchange como instrumento de cobertura de riesgo de precio para ganado bovino en Chile. Se buscará dar respuesta a las siguientes preguntas: ¿están integrados los mercados de ganado bovino chileno y el Chicago Mercantile Exchange?, ¿cuál es la cobertura óptima que minimiza el riesgo para un productor chileno que ingresa al mercado de futuros? Para ello, se utilizaron los precios al productor de ganado bovino en pie en el mercado nacional y los contratos futuros del Chicago Mercantile Exchange para el período comprendido entre enero de 1975 y marzo de 2012. A partir de un análisis econométrico, se determinó la existencia de integración y causalidad entre ambos precios (Dickey, Hasza \& Fuller, 1984; Hylleberg, Engle \& Granger, 1990) y la tasa de cobertura óptima (Working, 1953; Howard \& D'Antonio, 1984; Cecchetti, Cumby y Figlewski, 1988; Hsin, Kuo \& 
Troncoso y Cabas: Factibilidad del uso de contratos de futuros del Chicago Mercantile...

Lee, 1994; Cheng-Few, Kehluh \& Yang-Long, 2009) a través del Modelo de Johnson y Stein, que permitirá definir si es factible, desde un punto de vista económico, la cobertura con futuros en productores bovinos nacionales.

El presente trabajo se divide en cuatro apartados: en la sección I, se abordan brevemente algunos aspectos sobre la regulación, el funcionamiento y la evolución de este tipo de mercados en Chile; en la sección II, se describen los modelos econométricos clásicos para la estimación de ratios de cobertura óptima; en la sección III, se presentan y analizan los principales resultados obtenidos. Finalmente, se exponen las conclusiones más relevantes del trabajo.

\section{El mercado de futuros en Chile}

El éxito de los mercados futuros en Estados Unidos, desde su regulación en 1936 con la creación del Commodity Exchange Authority, elevó las expectativas sobre la creación de un mercado de futuros en Chile. Dadas las condiciones económicas nacionales e internacionales en la década de los 70, se pensó que tendría un éxito similar, pues Chile estaba viviendo un proceso de apertura económica y la problemática de la volatilidad de precios se había instaurado en los mercados internacionales. Así, en 1990 comenzó a funcionar en la Bolsa de Comercio de Santiago un mercado de contratos futuros sobre dólares, y a partir de 1991 los futuros iniciaron operaciones sobre el Índice de Precio Selectivo de Acciones (IPSA). Pese a todas las expectativas, el mercado de futuros en Bolsa no tuvo éxito en Chile y gozó de una efímera vida.

Una de las principales causas del fracaso del mercado de futuros fue la desinformación y la escasa educación de los inversionistas respecto del tema, quienes desconocían las ventajas de invertir en este tipo de mercados y optaron por especular con instrumentos ya probados. Esto, sumado al reducido tamaño del mercado nacional y a la excesiva regulación, provocó una falta de liquidez tan grande que desde 1998 no se intercambian estos instrumentos en la Bolsa de Comercio de Santiago. 
Paralelamente al fracaso del mercado de futuros en Chile, se han desarrollado, con relativo éxito, las negociaciones extrabursátiles (over the counter -OTC-) sobre instrumentos financieros (acciones, bonos, materias primas, swaps o derivados de crédito), directamente entre dos partes y fuera del ámbito de los mercados organizados. Lo que diferencia a estas operaciones es su carácter privado no estandarizado, cuyo contenido es determinado libremente y de común acuerdo. Sin embargo, las mayores dificultades de este tipo de mercados son su escasa liquidez y el alto riesgo de crédito, el cual se relaciona con la probabilidad de incumplimiento de las obligaciones emanadas de estos instrumentos, lo que aumenta los costos y limita el uso de derivados en empresas (principalmente exportadoras) que se ven afectadas por fluctuaciones del tipo de cambio o de algunos índices bursátiles.

\section{Modelos de cobertura óptima con futuros}

\section{A. Modelo de Johnson y Stein (JS)}

Johnson (1960) y Stein (1961) desarrollaron un modelo (JS) basado en la teoría de selección de carteras para determinar la razón de cobertura óptima de mínima varianza, es decir, la proporción de producto a cubrir en un mercado de derivados que minimiza el riesgo por volatilidad de precios.

El modelo JS define $Y$ como la cantidad de producto que se comercializa en el mercado de disponible y $F$ como la cantidad en el mercado de futuros, $P_{t}^{D}$ es el precio de disponible en el período final $t, P_{0}^{D}$ es el precio inicial de disponible, $P_{t}^{F}$ es el precio en el mercado de futuros en el período t y $P_{0} F$ es el precio de futuros inicial. Luego, la varianza del portafolio de un productor que transa en ambos mercados viene dada por:

$$
\sigma^{2}=Y^{2} \sigma_{P D}^{2}+F^{2} \sigma_{P F}^{2}+2 Y F \sigma_{P D, P F},
$$

donde $\sigma_{P D}^{2}$ y $\sigma_{P F}^{2}$ son las varianzas de los precios de disponible y futuros y $\sigma_{P D, P F}$ es la covarianza. La mínima varianza viene dada por la primera derivada de la ecuación (1) con respecto a la cantidad de producto en el mercado de futuros $(F)$ :

$$
C M V=-\frac{\sigma_{P D, P F}}{\sigma_{P F}^{2}}
$$


Troncoso y Cabas: Factibilidad del uso de contratos de futuros del Chicago Mercantile...

$C M V$ es la relación $(F / Y)$ y corresponde a la proporción de la producción total que debería cubrirse en el mercado de futuros y que minimiza el riesgo por fluctuación de precio. La expresión (2) se corresponde con la pendiente de la recta de regresión por mínimos cuadrados ordinarios (MCO) entre los cambios de los precios (o rendimientos) al contado respecto a los de futuro (Ederington, 1979), tal como se muestra a continuación:

$$
\Delta P_{t}^{D}=\alpha_{0}+\alpha_{1} \Delta P_{t}^{F}+\varepsilon_{t}
$$

Como las series de precios suelen comportarse de manera aleatoria, se pueden incorporar las primeras diferencias rezagadas de los precios como variables explicativas, a fin de obtener una mejor especificación del modelo (Arias \& Segura, 2000; Myers \& Thompson, 1989). Luego, la ecuación (3) se puede expresar de la siguiente forma:

$$
\Delta P_{t}^{D} \alpha_{0}+\alpha_{1} \Delta P_{t}^{F}+\alpha_{2} \Delta P_{t-1}^{D}+\alpha_{3} \Delta P_{t-1}^{F}+\varepsilon_{t} .
$$

En la ecuación (4) la constante $\left(\alpha_{0}\right)$ representa la base (diferencia entre precios de disponible y futuros) cuando el mercado de futuros es insesgado y los dos mercados convergen. La desviación estándar del término de error $\left(\varepsilon_{t}\right)$ corresponde al riesgo de base, es decir, aquel riesgo que no se puede solventar mediante la posición en el mercado de futuros.

El modelo propuesto por Johnson y Stein establece algunos supuestos bastante restrictivos: quienes participan en el mercado no tienen restricciones presupuestarias, costos financieros o comisiones de bolsa; los residuos del modelo de regresión no presentan problemas (autocorrelación o heterocedasticidad), y se asume que la variación en los rendimientos de los mercados sigue una distribución normal. De todas maneras, el modelo ha sido utilizado ampliamente, aunque algunos autores han propuesto métodos alternativos para minimizar el riesgo de la cartera.

\section{B. Ratio de cobertura de Sharpe}

Howard y D'Antonio (1984) propusieron la ratio de cobertura de Sharpe, que considera la trade-off entre riesgo y retorno. Según este enfoque, el objetivo 
de la cobertura es maximizar la rentabilidad "anormal" a la razón de riesgo de sus portafolios de contado (spot) y futuro, considerando que la tasa libre de riesgo existe, que el margen y los costos no se requieren, que el coeficiente de aversión al riesgo es desconocido y que no existen preferencias entre el contado y los activos libres de riesgo. Bajo esta ratio de cobertura se ha de maximizar la siguiente función llamada medida de riesgo-retorno de Sharpe:

$$
\theta=\frac{E\left(R_{h}\right)-R_{F}}{\sigma_{h}},
$$

donde $E\left(R_{h}\right)$ es el retorno esperado (porcentaje) del portafolio de contado (spot) y futuro, $R_{F}$ es el interés libre de riesgo (porcentaje) y $\sigma_{h}$ es la desviación estándar de los retornos del portafolio.

Siguiendo a Howard y D'Antonio (1984), $E\left(R_{h}\right)$ y $\sigma_{h}$ pueden ser especificados como:

$$
\begin{gathered}
E\left(R_{h}\right)=\frac{C_{s} P_{s} \bar{r}_{s}+C_{f} P_{f} \bar{r}_{f}}{C_{s} P_{s}}, \\
\sigma_{h}=\frac{1}{C_{s} P_{s}} \sqrt{C_{s}^{2} P_{s}^{2} \sigma_{s}^{2}+C_{f}^{2} P_{f}^{2} \sigma_{f}^{2}+2 C_{s} C_{f} P_{s} P_{f} \sigma_{s} \sigma_{f} \rho},
\end{gathered}
$$

donde, $C_{s}, C_{f}$ son el número de unidades de contado (spots) y de futuros, respectivamente; $P_{s}, P_{f}$ son el precio corriente por unidad de contado y de futuro, respectivamente; $\bar{r}_{s}, \bar{r}_{f}$ son el retorno esperado (un período) para contado y para futuro, respectivamente; $\sigma_{s} \sigma_{f}$ son la desviación estándar de los retornos de contado y de futuro, respectivamente, y $\rho$ es la correlación entre los retornos de contado y futuro.

Sustituyendo (6) y (7) en la ecuación (5), calculando la derivada de (5) con respecto a $C_{f}$ y reagrupando, se obtiene la ratio de cobertura óptima de Sharpe:

$$
C_{f}^{*}=-\frac{\left(\frac{\sigma_{s}}{\sigma_{f}}\right)\left[\frac{\sigma_{s}}{\sigma_{f}}\left(\frac{\bar{r}_{f}}{\bar{r}_{s}-R_{F}}\right)-\rho\right]}{\left[1-\frac{\sigma_{s}}{\sigma_{f}}\left(\frac{\bar{r}_{f} \rho}{\bar{r}_{s}-R F}\right)\right]} .
$$


Troncoso y Cabas: Factibilidad del uso de contratos de futuros del Chicago Mercantile...

Usando la ecuación (5) maximizada, Howard y D’Antonio derivaron una expresión para medir la efectividad de la cobertura $(H E)^{1}$ a través del cambio en el retorno con relación al interés libre de riesgo:

$$
H E=-\frac{\frac{\left[E\left(R_{h}\right)-R_{F}\right]}{\sigma_{h}}}{\frac{\left[\bar{r}_{s}-R_{F}\right]}{\sigma_{s}}} .
$$

\section{Ratio de cobertura de media varianza}

La ventaja de este método de cálculo de cobertura es su simplicidad: asume que todos los inversionistas se focalizan solamente en la minimización del riesgo. Hsin et al. (1994) estimaron la ratio de cobertura a través de la maximización de la siguiente función de utilidad:

$$
V\left(E\left(R_{h}\right), \sigma, A\right)=E\left(R_{h}\right)-0,5 A \sigma_{h}^{2},
$$

donde $A$ es el coeficiente de aversión al riesgo del inversionista, $E\left(R_{h}\right)$ es la esperanza del rendimiento del portafolio compuesto por posiciones en contado y futuro y $\sigma_{h}^{2}$ es la varianza del portafolio.

El coeficiente de aversión al riesgo representa el grado de influencia del riesgo sobre la utilidad individual. Comúnmente, la mayoría de los cobertores son aversos al riesgo con un coeficiente $A$ positivo.

La ratio de cobertura óptima de media varianza, derivado desde la diferencial de primer orden de la función de utilidad es:

$$
h=\frac{C_{f}^{*}}{C_{s}}=-\left[\frac{\bar{r}_{f}}{A \sigma_{f}^{2}}-\rho \frac{\sigma_{s}}{\sigma_{f}}\right],
$$

donde los subíndices $f$ y $s$ hacen referencia a futuros y contado $(s p o t) . E\left(R_{f}\right)$ se calcula a partir de la media muestral de los retornos, la desviación estándar es estimada a partir del error estándar muestral y el coeficiente de correlación se calcula desde el coeficiente de correlación muestral (Cheng-Few et al., 2009).

Para $E\left(R_{s}\right)-R_{F} \neq 0$ 
La efectividad de la cobertura de media varianza se puede evaluar por el incremento en la función de utilidad después de la cobertura:

$$
H E=V *-V
$$

donde $V *$ es la utilidad después de la cobertura y $V$ la utilidad antes de ella. Si la cobertura cumple el objetivo para el que se tomó, es decir, es efectiva, $H E$ será positivo; de lo contrario, será negativo. La utilización de este método para el cálculo de cobertura óptima concuerda con la teoría económica; sin embargo, en la práctica, la función de utilidad del inversor es específica y resulta difícil estimar una medida de aversión al riesgo, pues a partir de este método podría resultar negativa.

\section{Estimación de cobertura óptima con futuros}

\section{A. Método}

Para estimar la tasa óptima de cobertura con contratos de futuros, en primer lugar, se practicó un análisis descriptivo de las series temporales para determinar, a priori, la posible presencia de estacionalidad y estacionariedad en los datos. Como segundo paso, se determinó la existencia de una relación de causalidad entre los precios de futuros en el Chicago Mercantile Exchange y los precios al productor de novillo en la feria ganadera Tattersall de Santiago. Una vez concluido lo anterior, se aplicaron test de raíces unitarias a las frecuencias cero y estacionales (bimensual, períodos de cuatro meses, trimestral, semestral, frecuencia $5 \pi / 6$ y raíz unitaria anual) para diagnosticar estadísticamente la estacionariedad o el orden de integración I(d) de ambas series (Soto \& Tapia, 2000). Una vez determinado el orden de integración de los datos, se crearon vectores de cointegración en las distintas frecuencias y se estimó por mínimos cuadrados ordinarios el modelo de corrección de errores (MCE), basado en Johnson y Stein (1960), que mejor cumple las condiciones de estabilidad. Finalmente, y a partir del MCE, se determinó el óptimo de cobertura para productores nacionales y para las elasticidades de transmisión de precios entre ambos mercados. 
Troncoso y Cabas: Factibilidad del uso de contratos de futuros del Chicago Mercantile...

\section{B. Datos}

Los precios de contado para ganado bovino vivo fueron extraídos del portal de la Oficina de Estudios y Políticas Agrarias del Ministerio de Agricultura de Chile (ODEPA), y corresponden a precios al productor de novillo por kilo sin IVA y deflactados por IPC de marzo de 2012. El período de análisis va de enero de 1975 a marzo de 2012.

Los futuros fueron obtenidos del sitio web del Chicago Mercantile Exchange y comprenden el mismo período antes señalado. Dado que se reportan las cotizaciones diarias, se utilizó para cada mes el precio de cierre (medido en centavos de dólar por libra) del último día hábil para el contrato de futuros más cercano. Esta serie de tiempo fue llevada a pesos chilenos utilizando los tipos de cambio mensuales del Banco Central de Chile y deflactada por IPC a marzo de 2012.

Para facilitar el análisis y comprensión, se decidió convertir ambas series a precios por unidad animal bovina, es decir, 450 kilos, que es el peso estándar de venta de los novillos gordos en las ferias ganaderas de Chile. Al estimar las relaciones de cointegración, las series en cada frecuencia fueron suavizadas utilizando logaritmos naturales.

\section{Análisis de estacionalidad en las series precio disponible real (PDR) $y$ precio futuro real (PFR)}

\section{Gráficos de las series y subseries estacionales}

Los gráficos anuales de las series (Gráfico 1) muestran cierta estacionalidad que se repite año a año en los precios disponibles, presentando los niveles más altos en septiembre y los menores precios en mayo, aunque esto último no es tan evidente como en el primer caso. Las décadas de los 70 y 80 son las de mayor volatilidad, y a partir de 1990 los precios se estabilizan, aunque persisten algunos picos como septiembre de 1997 y agosto de 2008, ambos períodos en los que la economía nacional se veía afectada por crisis económicas internacionales. 
Gráfico 1. Series anuales de PDR y PFR

Precios disponibles (pesos reales)

Precios futuros (pesos reales)

1975-1981
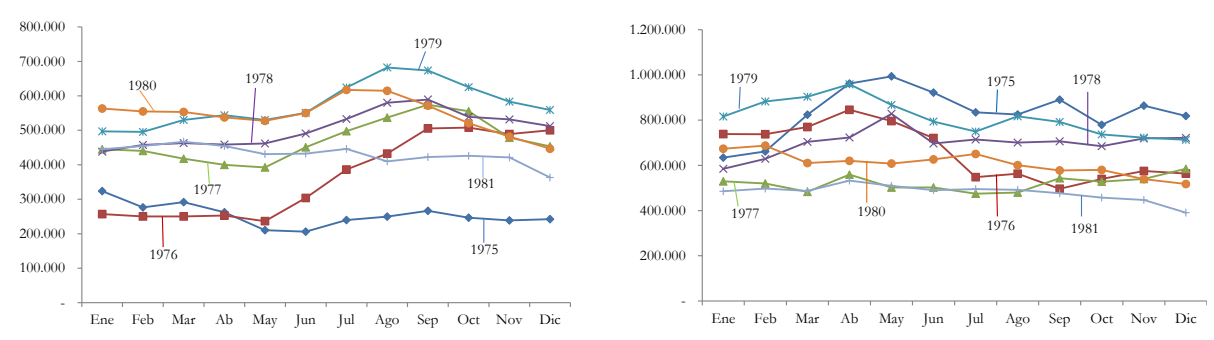

1982-1988
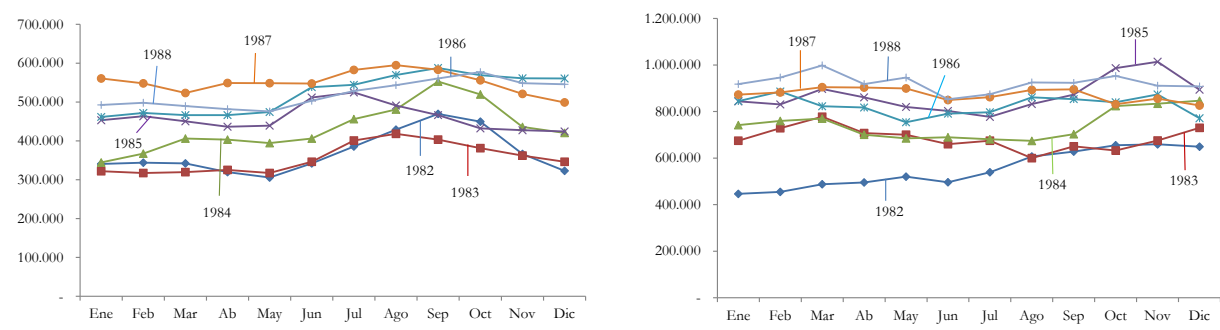

1989-1995
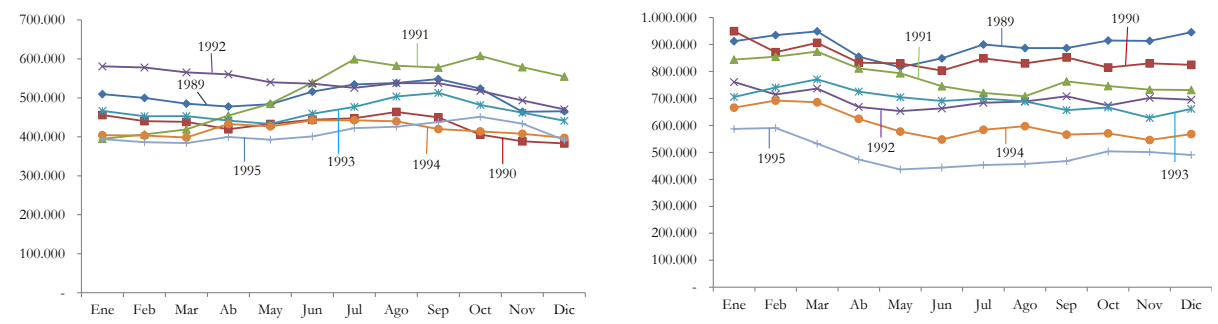

1996-2002
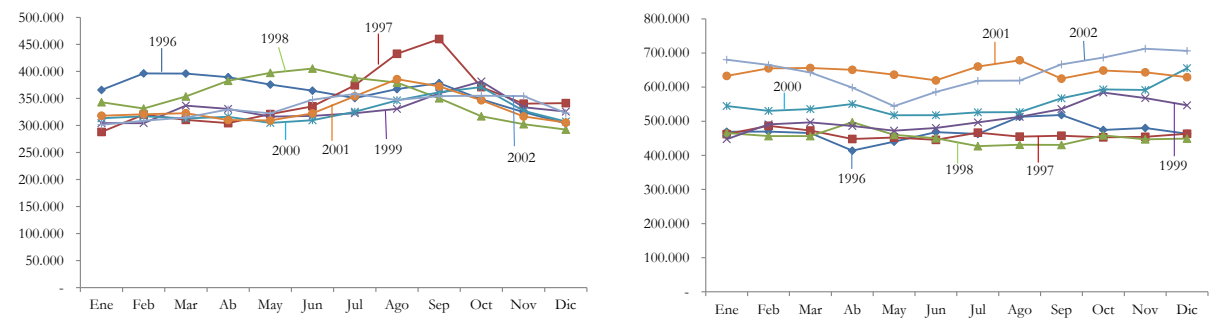

Continúa

Lecturas de Economía -Lect. Econ. - No. 90. Medellín, enero-junio 2019 
Troncoso y Cabas: Factibilidad del uso de contratos de futuros del Chicago Mercantile...

Figura 1. Continuación

Precios disponibles (pesos reales)

Precios futuros (pesos reales)

2003-2009
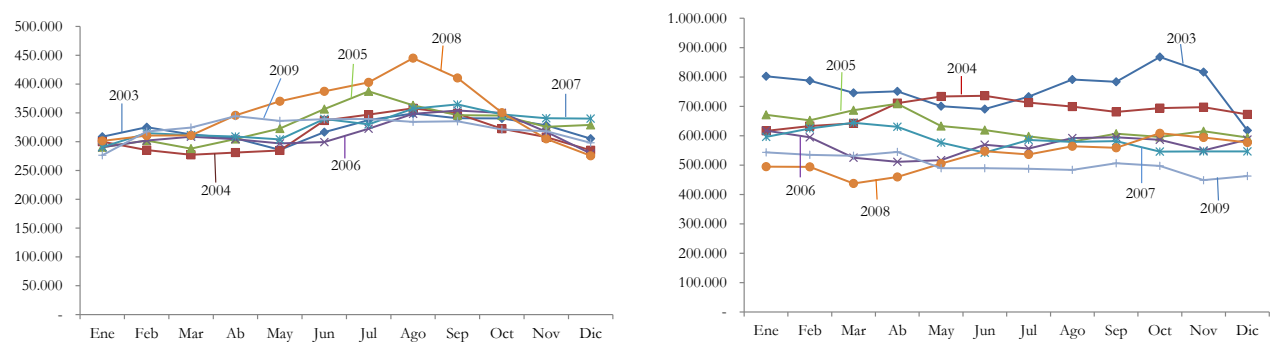

2010-2012
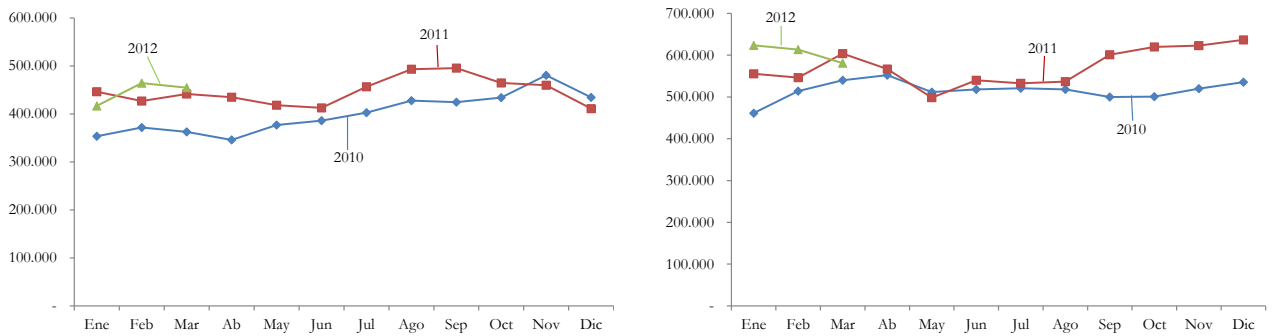

Fuente: elaboración propia.

Los futuros no muestran un comportamiento estacional que sea identificable a primera vista. En general pareciera que los meses de marzo y abril muestran, en promedio, los mayores precios, aunque se torna difuso en la medida que pasan los años y los mayores precios se trasladan a los meses de octubre, noviembre y diciembre, lo que cobra sentido dadas las festividades propias en dichos períodos.

Una herramienta más útil para apreciar el comportamiento de los precios en cada mes es utilizar gráficos de líneas apiladas, como los que se muestran en el Gráfico 2. El mes de septiembre es el que ostenta los mayores precios disponibles, y se aprecia un patrón estacional entre los meses de junio y noviembre de cada año. En los futuros también se confirma la hipótesis inicial (marzo y abril son los meses con los precios más altos), aunque todavía no es posible tener certeza de algún patrón estacional claramente definido, haciéndose necesaria la aplicación de pruebas más formales. 
Gráfico 2. Lineas apiladas de las series PDR y PFR

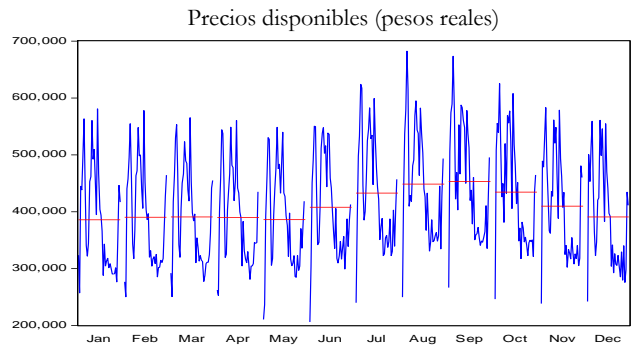

Precios futuros (pesos reales)

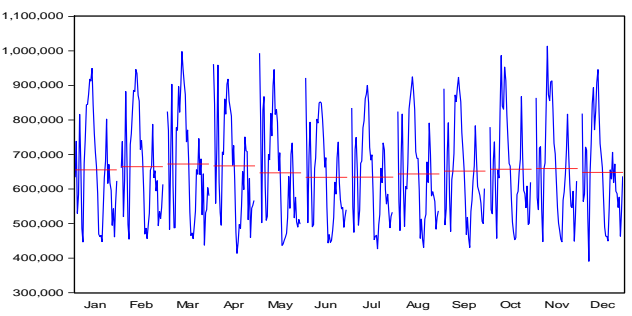

Fuente: elaboración propia.

\section{X-12-Arima}

Para la detección de estacionalidad, se determinó el tipo de esquema estacional $^{2}$ que más se ajusta a cada serie por medio del análisis de gráficos media/desviación típica y de la relación entre coeficientes de variación de las diferencias y cocientes de primer orden (Peña, 2010).

Como se aprecia en los diagramas de dispersión (Gráfico 3), persiste una relación positiva entre las medias y desviaciones típicas que a simple vista denota la presencia de un esquema estacional del tipo multiplicativo.

Gráfico 3. Medialdesviación típica de las series PDR y PFR
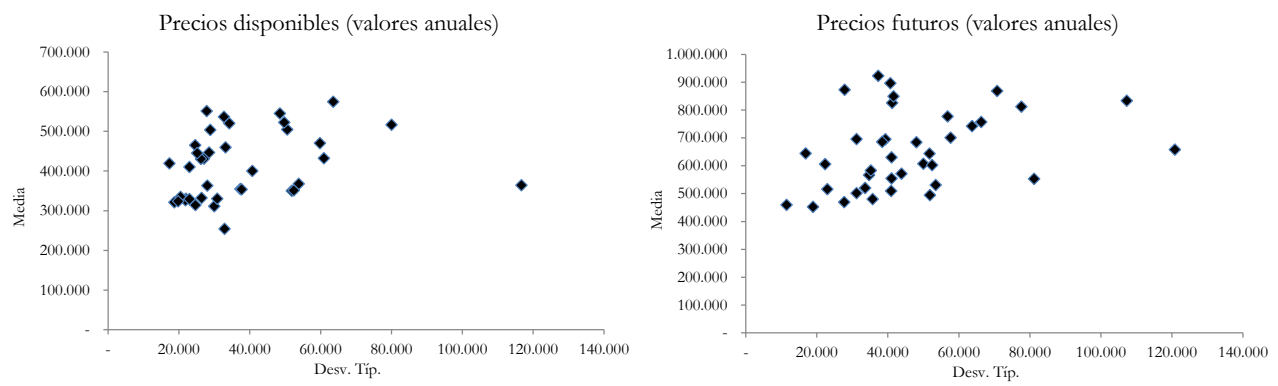

Fuente: elaboración propia.

2 Multiplicativo $=T_{t} x C_{t} x S_{t} x I_{t}$, Aditivo $=T_{t}+C_{t}+S_{t}+I_{t}$ y Seudo Aditivo $=T_{t} C_{t}\left(S_{t}+\right.$ $\left.I_{t}-1\right)$. 
Troncoso y Cabas: Factibilidad del uso de contratos de futuros del Chicago Mercantile...

Tal como se observa en la Tabla 1, el método de coeficientes de variación (CV) corrobora la primera impresión sobre el esquema estacional, pues en los precios disponibles y futuros los coeficientes de las primeras diferencias son mayores a los coeficientes de los cocientes (en términos absolutos).

Tabla 1. Coeficientes de variación de las series PDR y PFR

\begin{tabular}{ccc}
\hline Coeficiente & \multicolumn{2}{c}{ Valores } \\
\hline $\mathrm{CV}(\mathrm{D})$ & 13,1342 & $-15,6719$ \\
$\mathrm{CV}(\mathrm{K})$ & 0,1902 & 0,1722 \\
\hline
\end{tabular}

Fuente: elaboración propia.

Conocido el esquema estacional de las series, se les aplicó el método X12-ARIMA para diagnosticar la presencia de estacionalidad siguiendo las recomendaciones de Gallardo y Rubio (2009). Eviews ofrece cuatro pruebas que muestran la serie original ajustada por el efecto calendario ${ }^{3}$ y sin el componente de ciclo-tendencia. La Tabla 2 resume los estadísticos asociados a cada prueba de estacionalidad.

Tabla 2. Pruebas de estacionalidad X-12-ARIMA

\begin{tabular}{lcc}
\hline Prueba o contraste & $\begin{array}{c}\text { Precios disponibles } \\
\text { estadístico }\end{array}$ & $\begin{array}{c}\text { Precios futuros } \\
\text { estadístico }\end{array}$ \\
\hline Prueba F de estacionalidad estable & $68,866^{* *}$ & $5,481^{* *}$ \\
Prueba no paramétrica de Kruskal-Wallis & $297,8809^{* *}$ & $71,2232^{* *}$ \\
Contraste de estacionalidad móvil & $3,766^{* *}$ & $2,552^{* *}$ \\
Contraste combinado de estacionalidad identificable & 0,3645 & 1,1563 \\
\hline
\end{tabular}

Nota: ${ }^{* *}$ valores muy significativos $\mathrm{p}<0,01$.

Fuente: elaboración propia.

El estadístico F de la prueba de estacionalidad estable resultó estadísticamente significativo al $1 \%$, lo que rechaza la hipótesis nula que afirma que las

3 A excepción de los casos en los que el ajuste estacional se realiza sin estimar el efecto calendario. 
medias de los 38 años (1975-2012) son iguales y permite suponer la existencia de estacionalidad estable. Como esta prueba supone normalidad del componente irregular, la no existencia de ella hace que el estadístico pierda validez y que sea necesaria la aplicación de otras pruebas para corroborar la evidencia.

El estadístico de la prueba no paramétrica de Kruskal-Wallis es significativo al $1 \%$ en ambos casos; esto rechaza la hipótesis nula de igualdad de medias y permite identificar estacionalidad.

Finalmente, el contraste de estacionalidad móvil arroja valores estadísticamente significativos que rechazan la hipótesis nula que afirma que el efecto de todos los años es el mismo y, por lo tanto, se asume la existencia de estacionalidad móvil.

\section{Identificación de estacionariedad}

El Gráfico 4 presenta la evolución de los precios disponibles y futuros reales para la muestra en análisis. Es evidente que ambas series presentan un comportamiento procíclico coincidente a lo largo del tiempo.

Gráfico 4. Evolución de precio futuro y disponible real de ganado bovino, 1975-2012

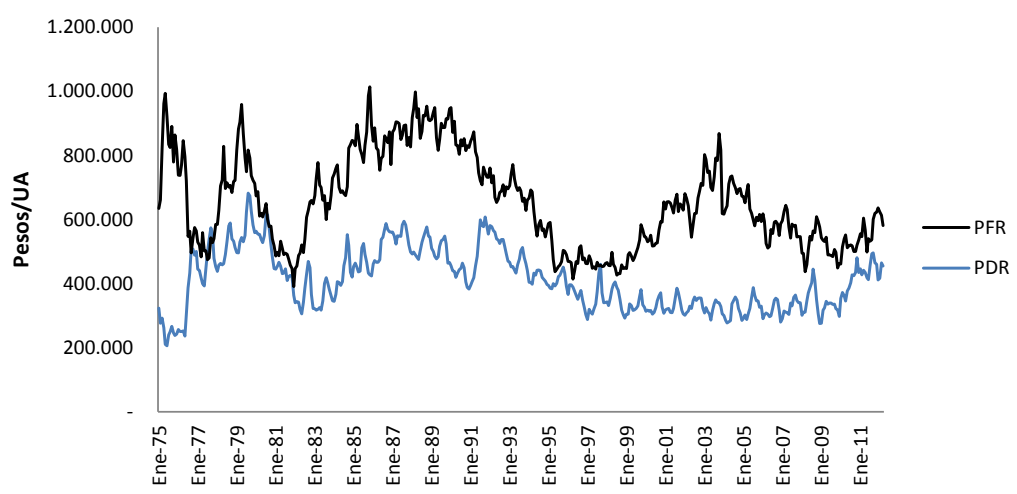

Fuente: elaboración propia con base en datos del Chicago Mercantile Exchange y ODEPA.

A primera vista, las series no presentan patrones que hagan suponer medias y varianzas constantes, y siguen una leve tendencia a la baja. Para 
Troncoso y Cabas: Factibilidad del uso de contratos de futuros del Chicago Mercantile...

complementar el diagnóstico de estacionariedad en las series, se estimaron los coeficientes de autocorrelación simple y parcial presentados en las Tablas 3 y 4.

Tabla 3. Correlograma de la serie PDR

\begin{tabular}{|c|c|c|c|c|c|c|}
\hline Autocorrelación & A. Parcial & Rezagos & $\mathrm{AC}$ & ACP & Est-Q & Probabilidad \\
\hline.||$^{* * * * * * *}$ &.$\left.\right|^{* * * * * * *}$ & 1 & 0,9610 & 0,9610 & 415,9 & 0,0000 \\
\hline.||$^{* * * * * * \mid}$ & $* * *||$. & 2 & 0,8950 & $-0,3820$ & 777,3 & 0,0000 \\
\hline.$\left.\right|^{* * * * * * \mid}$ & $.\left.\right|^{*} \mid$ & 3 & 0,8320 & 0,1480 & $1.089,9$ & 0,0000 \\
\hline$.||^{* * * * * *} \mid$ &.$||$. & 4 & 0,7800 & 0,0560 & $1.365,3$ & 0,0000 \\
\hline$\cdot||^{* * * * *} \mid$ & $.\left.\right|^{*} \mid$ & 5 & 0,7420 & 0.0960 & $1.615,5$ & 0,0000 \\
\hline$.||^{* * * * *} \mid$ &. $\mid 1$ & 6 & 0,7170 & 0,0680 & $1.849,7$ & 0,0000 \\
\hline$.||^{* * * * *} \mid$ &.$||$. & 7 & 0,7000 & 0,0350 & $2.072,9$ & 0,0000 \\
\hline$.||^{* * * * *} \mid$ & $.\left.\right|^{*} \mid$ & 8 & 0,6930 & 0,1620 & $2.292,6$ & 0,0000 \\
\hline$.||^{* * * * *} \mid$ & $.\left.\right|^{*} \mid$ & 9 & 0,6990 & 0,1160 & $2.516,2$ & 0,0000 \\
\hline||$^{* * * * *} \mid$ &.$||$. & 10 & 0,7090 & 0,0470 & $2.746,9$ & 0,0000 \\
\hline · & . & . & · & . & . & . \\
\hline · & · & · & · & . & $\cdot$ & · \\
\hline$\cdot$ & · & . & . & . & · & . \\
\hline$.\left.\right|^{* *} \mid$ & $.\left.\right|^{*} \mid$ & 34 & 0,3010 & 0,0820 & $5.013,5$ & 0,0000 \\
\hline$.\left.\right|^{* *} \mid$ &.$||$. & 35 & 0,3110 & 0,0360 & $5.060,5$ & 0,0000 \\
\hline$.\left.\right|^{* *} \mid$ &.$||$. & 36 & 0,3020 & 0,0510 & $5.104,9$ & 0,0000 \\
\hline
\end{tabular}

Fuente: elaboración propia.

Los coeficientes de autocorrelación simple de ambas series son estadísticamente significativos hasta el rezago de orden 36 , son positivos y se encuentran fuera del intervalo de confianza del $95 \%$. Pese a que el nivel de las autocorrelaciones tiende a disminuir, no existe evidencia estadística para no rechazar la hipótesis individual de que cada $\rho_{k}=0$, ya que ninguno de los intervalos contiene el valor de cero, sugiriendo que las series PDR y PFR son no estacionarias. 
Tabla 4. Correlograma de la serie PFR

\begin{tabular}{ccccccc}
\hline.$\left.\right|^{* * * * * * *}$ &.$\left.\right|^{* * * * * * *}$ & 1 & 0,9610 & 0,9610 & 415,3 & 0,0000 \\
.$\left.\right|^{* * * * * * *}$ &.$||$. & 2 & 0,9210 & $-0,0290$ & 797,5 & 0,0000 \\
.$\left.\right|^{* * * * * *}$ &.$||$. & 3 & 0,8790 & $-0,0440$ & $1.146,5$ & 0,0000 \\
.$\left.\right|^{* * * * * \mid}$ & $.\left.\right|^{*} \mid$ & 4 & 0,8510 & 0,1550 & $1.474,2$ & 0,0000 \\
.$\left.\right|^{* * * * * \mid}$ & $.\left.\right|^{*} \mid$ & 5 & 0,8330 & 0,1220 & $1.789,3$ & 0,0000 \\
.$\left.\right|^{* * * * * \mid}$ &.$||$. & 6 & 0,8190 & 0,0310 & $2.094,7$ & 0,0000 \\
.$\left.\right|^{* * * * * \mid}$ & $*||$. & 7 & 0,7990 & $-0,0810$ & $2.385,6$ & 0,0000 \\
.$\left.\right|^{* * * * * \mid}$ &.$||$. & 8 & 0,7780 & 0,0400 & $2.662,6$ & 0,0000 \\
$.\left.\right|^{* * * * *} \mid$ &.$||$. & 9 & 0,7580 & 0,0300 & $2.926,0$ & 0,0000 \\
$.\left.\right|^{* * * * *} \mid$ &.$||$. & 10 & 0,7410 & 0,0180 & $3.178,4$ & 0,0000 \\
. &. &. &. &. &. &. \\
. &. &. &. &. &. &. \\
. &. &. &. &. &. &. \\
$.\left.\right|^{* *} \mid$ &.$||$. & 34 & 0,3500 & 0,0740 & $5.952,1$ & 0,0000 \\
$.\left.\right|^{* *} \mid$ &.$||$. & 35 & 0,3500 & 0,0190 & $6.011,9$ & 0,0000 \\
$.\left.\right|^{* *} \mid$ &.$||$. & 36 & 0,3450 & $-0,0590$ & $6.070,1$ & 0,0000 \\
\hline
\end{tabular}

Fuente: elaboración propia.

Los coeficientes de autocorrelación parcial de PDR para los rezagos de orden 1 y 2 son los de mayor magnitud. La serie PFR presenta alta correlación positiva con el primer rezago $\left(\rho_{1}=0,961\right)$, y también se ven otros coeficientes estadísticamente significativos, pero sus magnitudes no superan $\pm 0,16$.

1. Pruebas de raíz unitaria en frecuencia cero y orden de integración de las series

Gracias al análisis de los correlogramas, se obtuvo una aproximación del orden de integración de los precios de disponible y futuros; sin embargo, es imperante corroborar esta intuición usando pruebas de raíz unitaria que permitan contrastar la hipótesis de no estacionariedad en niveles. En la Tabla 5 se muestran los resultados de las pruebas de Dickey-Fuller (DF), Dickey-Fuller 
Troncoso y Cabas: Factibilidad del uso de contratos de futuros del Chicago Mercantile...

Aumentada (DFA), Phillips-Perron (PP) y Kwiatkowski-Phillips-SchmidtShin (KPSS) ${ }^{4}$ para los modelos con o sin intercepto y tendencia.

Tabla 5. Resumen de las pruebas básicas de raiz unitaria

\begin{tabular}{|c|c|c|c|c|c|c|c|c|c|c|c|c|}
\hline & & \multicolumn{2}{|c|}{ DF } & \multicolumn{2}{|c|}{ DFA } & \multicolumn{2}{|c|}{ PP } & \multicolumn{5}{|c|}{ KPSS } \\
\hline & $\begin{array}{c}\phi_{1}=\mathrm{No}_{\mathrm{o}} \\
t=\mathrm{No}_{\mathrm{o}}\end{array}$ & $\begin{array}{l}\phi_{1}=\mathrm{Si} \\
t=\mathrm{No}\end{array}$ & $\begin{array}{c}\phi_{1}=\mathrm{Si} \\
t=\mathrm{Si}\end{array}$ & $\begin{array}{c}\phi_{1}=\mathrm{No}_{\mathrm{o}} \\
t=\mathrm{No}_{\mathrm{o}}\end{array}$ & $\begin{array}{c}\phi_{1}=\mathrm{Si} \\
t=\mathrm{No}_{\mathrm{o}}\end{array}$ & $\begin{array}{c}\phi_{1}=\mathrm{Si} \\
t=\mathrm{Si}\end{array}$ & Rezagos & $\begin{array}{c}\phi_{1}=\mathrm{No}_{\mathrm{o}} \\
t=\mathrm{No}_{\mathrm{o}}\end{array}$ & $\begin{array}{c}\phi_{1}=\mathrm{Si} \\
t=\mathrm{No}\end{array}$ & $\begin{array}{c}\phi_{1}=\mathrm{Si} \\
t=\mathrm{Si}\end{array}$ & $\begin{array}{c}\phi_{1}=\mathrm{Si} \\
t=\mathrm{No}_{\mathrm{o}}\end{array}$ & $\begin{array}{c}\phi_{1}=S \mathrm{~S} \\
t=\mathrm{Si}\end{array}$ \\
\hline PDR & $-0,42$ & $-2,97$ & $-3,33$ & $-0,25$ & $-3,74$ & $-4,27$ & $4,2,2$ & $-0,23$ & $-2,77$ & $-3,05$ & 0,88 & 0,19 \\
\hline PFR & $-0,70$ & $-2,96$ & $-3,29$ & $-0,70$ & $-2,96$ & $-3,29$ & $0,0,0$ & $-0,55$ & $-2,88$ & $-3,29$ & 0,67 & 0,16 \\
\hline $\begin{array}{l}\text { Valores } \\
\text { críticos } \\
\text { al } 95 \%\end{array}$ & $-1,94$ & $-2,87$ & $-3,42$ & $-1,94$ & $-2,87$ & $-3,42$ & - & $-1,94$ & $-2,87$ & $-3,42$ & 0,46 & 0,15 \\
\hline
\end{tabular}

Nota: $\phi_{1}=$ Intercepto y $t=$ Tendencia. Para determinar los rezagos óptimos de la prueba DFA se utilizó el criterio de Schwartz (bic).

Fuente: elaboración propia.

Como se aprecia en la Tabla 5, al contrastar los estadísticos de cada una de las pruebas de raíz unitaria para los modelos con y sin intercepto y tendencia con los valores críticos de MacKinnon (1991) es posible concluir que ambas series en niveles presentan, al menos, una raíz unitaria en la frecuencia cero, aunque es importante señalar que este tipo de pruebas suelen presentar inconsistencias causadas por su bajo poder cuando el verdadero proceso que siguen las variables es arbitrariamente cercano a una raíz unitaria (Cochrane, 1991). Esto ocurre, también, porque estas pruebas (incluyendo PP y KPSS) evalúan raíces unitarias en la frecuencia cero de las series y pasan por alto la existencia de estacionalidad. Para asegurar el orden de integración de ambas series, una práctica comúnmente aceptada consiste en analizar patrones estacionarios a través de gráficos y en aplicar la prueba DF a las series transformadas. En el Gráfico 5 se muestra el comportamiento de los precios disponibles y reales, una vez diferenciados.

Como se puede entrever, las gráficas sugieren que ambas series son estacionarias si se trabajan en primeras diferencias. Con la finalidad de tener una mayor certeza, en la Tabla 6 se exponen los resultados de la prueba DF.

4 La hipótesis nula de esta prueba supone la existencia de estacionariedad. 
Gráfico 5. Series en primeras diferencias
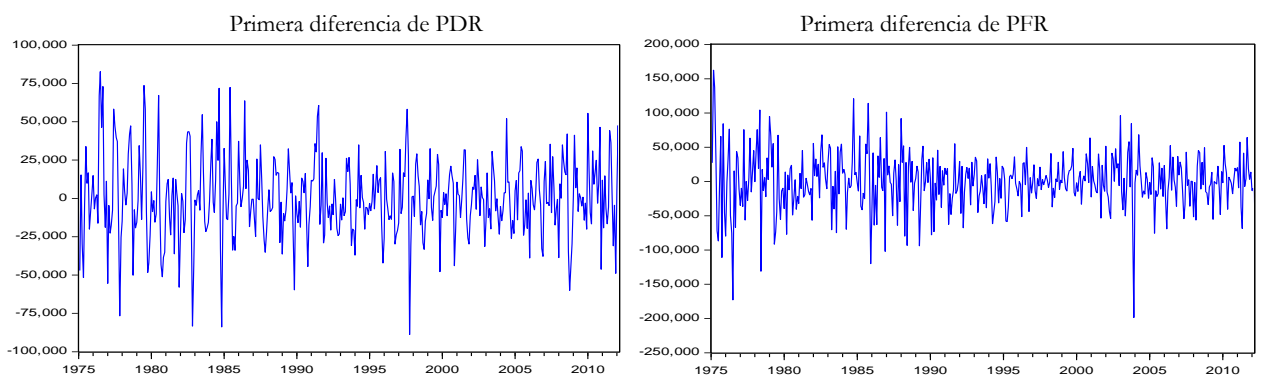

Fuente: elaboración propia.

Tabla 6. Estadístico de la prueba Dickey-Fuller con series en primeras diferencias

\begin{tabular}{ccc}
\hline & DPDR & DPFR \\
\hline Estadístico DF & $-14,6572$ & $-20,8063$ \\
Valores críticos & & \\
$1 \%$ & $-2,5702$ & $-2,5702$ \\
$5 \%$ & $-1,9415$ & $-1,9415$ \\
$10 \%$ & $-1,6162$ & $-1,6162$ \\
Durbin Watson & 1,8623 & 1,9758 \\
\hline
\end{tabular}

Fuente: elaboración propia.

Los resultados del análisis de raíz unitaria muestran que el estadístico de la prueba DF es superior a los valores críticos de MacKinnon (1991) al $1 \%$ de significancia; además, los estadísticos de Durbin-Watson rechazan la presencia de autorrelación residual de orden 1, lo que permite concluir que las series PDR y PFR en primeras diferencias son estacionarias e integradas del mismo orden al menos en la frecuencia cero.

\section{Pruebas de raices unitarias estacionales}

El análisis de estacionalidad practicado mediante métodos gráficos (Gráficos 1 y 2) y con la metodología X-12-ARIMA (ver Tabla 2) mostró la presencia de componentes estacionales en las series, lo cual sugiere la probable existencia de raíces unitarias en otras frecuencias. Para evaluar esto, se 
Troncoso y Cabas: Factibilidad del uso de contratos de futuros del Chicago Mercantile...

implementó la prueba de Hylleberg et al. (1990) (HEGY) utilizando la versión del modelo que considera constante, tendencia y variables dummies mensuales. El número de rezagos óptimos (p) de la prueba se definió usando el criterio de Schwartz (bic), a fin de mantener coherencia con las pruebas convencionales de raíz unitaria. Los resultados de la estimación se muestran en la Tabla 7:

Tabla 7. Prueba HEGY de series en niveles

Hipótesis nula: raíz unitaria

Hipótesis alternativa: estacionariedad

\begin{tabular}{lcccc}
\hline \multirow{2}{*}{ Frecuencia } & \multicolumn{2}{c}{ Estadístico } & \multicolumn{2}{c}{ Valor-p } \\
\cline { 2 - 5 } & PDR & PFR & PDR & PFR \\
\hline No estacional & $-3,3400$ & $-2,3350$ & 0,0500 & 0,1000 \\
Bimensual & $-4,0290$ & $-5,2610$ & 0,0100 & 0,0100 \\
Períodos de cuatro meses & 31,1110 & 60,7790 & 0,1000 & 0,1000 \\
Trimestral & 23,5110 & 35,6140 & 0,1000 & 0,1000 \\
Semestral & 36,8260 & 40,0560 & 0,1000 & 0,1000 \\
Frecuencia $5 \pi / 6$ & 24,3400 & 40,6390 & 0,1000 & 0,1000 \\
Anual & 41,9120 & 36,3940 & 0,1000 & 0,1000 \\
\hline
\end{tabular}

Fuente: elaboración propia.

$\mathrm{Al}$ analizar los resultados de la prueba, es factible concluir que en ambas series solo se rechaza la hipótesis nula de raíz unitaria bimensual $\left(H_{B}\right)$; dicho de otro modo, existen raíces unitarias en las frecuencias no estacional, cuatrimestral, trimestral, semestral, $5 p / 6$ y anual, siendo las series integradas estacionalmente del mismo orden.

Una vez definido el orden de integración estacional de las series, se implementó la prueba HEGY a las series diferenciadas estacionalmente para determinar si resultan estacionarias ${ }^{5}$. Los resultados de la prueba se presentan en la Tabla 8.

Los valores-p muestran con claridad que las series transformadas (PDRS y PFRS) son ahora estacionarias (al $5 \%$ de significancia) en todas las

5 Para mayor detalle véase Hylleberg et al. (1990). 
frecuencias estacionales. Además, los resultados de la prueba se verifican por medio de la inspección gráfica de las series diferenciadas estacionalmente, según se aprecia en el Gráfico 6.

Tabla 8. Prueba HEGY en series en primeras diferencias

\begin{tabular}{lcccc}
\hline $\begin{array}{l}\text { Hipótesis nula: raíz unitaria } \\
\text { Hipótesis alternativa: estacionariedad }\end{array}$ & \multicolumn{2}{c}{ Valor-p } \\
\hline \multirow{2}{*}{ Frecuencia } & \multicolumn{2}{c}{ Estadístico } & PDRS & PFRS \\
\cline { 2 - 5 } & PDRS & $-2,9500$ & 0,0100 & 0,0100 \\
No estacional & $-4,5200$ & $-2,3800$ & 0,0100 & 0,0160 \\
Bimensual & $-3,5010$ & 119,8640 & 0,0100 & 0,0100 \\
Períodos de cuatro meses & 115,1390 & 96,8270 & 0,0100 & 0,0100 \\
Trimestral & 138,8130 & 99,3490 & 0,0100 & 0,0100 \\
Semestral & 125,8480 & 88,9380 & 0,0100 & 0,0100 \\
Frecuencia 5 $/ 6$ & 99,8960 & 97,3430 & 0,0100 & 0,0100 \\
Anual & 119,3560 & & & \\
\hline
\end{tabular}

Fuente: elaboración propia.

Gráfico 6. Series transformadas estacionariamente
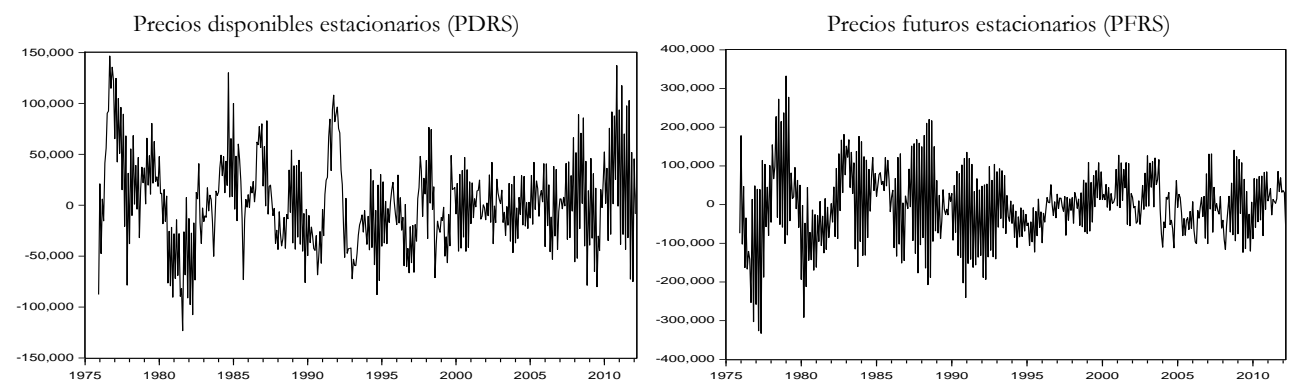

Fuente: elaboración propia.

\section{Prueba de causalidad de Granger}

Para determinar la presencia de una relación de causalidad entre ambas series, se implementó una prueba de Granger, la cual consiste en comprobar si los resultados o realizaciones de una variable sirven para predecir el comportamiento de otra y si esta relación tiene un carácter unidireccional o 
Troncoso y Cabas: Factibilidad del uso de contratos de futuros del Chicago Mercantile...

bidireccional. En la Tabla 9 se muestran los estadísticos de prueba y las respectivas probabilidades o valores-p.

Tabla 9. Prueba de causalidad de Granger

\begin{tabular}{cccc}
\hline Hipótesis Nula & Observaciones & Estadístico F & Valor-p \\
\hline PFR no Granger causa PDR & 445 & 7,9283 & $0,0004^{* * *}$ \\
PDR no Granger causa PFR & & 1,5338 & 0,2169 \\
\hline
\end{tabular}

Nota: ${ }^{* * *}$ valor altamente significativo $\mathrm{p}<0,001$.

Fuente: elaboración propia.

Los valores-p permiten corroborar la existencia de una relación de causalidad unidireccional entre ambas series de precios, en el sentido de que son los precios de futuro los que causan los precios disponibles y no a la inversa, resultado que es acorde con lo esperado.

\section{E. Análisis de cointegración estacional y modelo de corrección de errores}

Las pruebas de raíces unitaria convencionales y HEGY, expuestas en los apartados precedentes, mostraron que las series son no estacionarias en niveles, pero que al diferenciarlas estacionalmente resultan integradas del mismo orden. Este resultado conduce a evaluar la existencia de una relación de largo plazo o de cointegración por medio de una combinación lineal estacionaria $(\mathrm{I}(0))$ que asegure una relación de equilibrio entre las series originales para un cierto nivel de significancia (Granger \& Newbold, 1974; Granger, 1980; Engle \& Granger, 1987).

La evaluación de la existencia de cointegración estacional en las frecuencias cero, cuatrimestral, trimestral, semestral, $5 p / 6$ y anual se realizó en dos etapas. En primer lugar, se determinó el número de vectores de cointegración en cada frecuencia usando la prueba de traza propuesta por Johansen \& Juselius (1990). En la segunda etapa, se estimó cada uno de los vectores de cointegración y el modelo de corrección de errores para asegurar la estabilidad en el largo plazo. En la Tabla 10 se muestran los vectores estimados en cada frecuencia, de acuerdo con la metodología Johansen. 
Tabla 10. Vectores de cointegración estacional

\begin{tabular}{lccc}
\hline & & Vector de cointegración & Error estándar \\
\hline Variable dependiente & LPDRkt & 1 & - \\
\hline Frecuencia & & & \\
\hline Cero (largo plazo) & LPFR1t & $-0,9671$ & $(0,0034)$ \\
Cuatrimestral (1) & LPFR3t & $-0,6447$ & $(0,1353)$ \\
Cuatrimestral (2) & LPFR4t & $-0,8067$ & $(0,1641)$ \\
Trimestral (1) & LPFR5t & $-0,7875$ & $(0,1445)$ \\
Trimestral (2) & LPFR6t & $-0,8751$ & $(0,1764)$ \\
Semestral (1) & LPFR7t & $-1,0594$ & $(0,2466)$ \\
Semestral (2) & LPFR8t & $-0,9683$ & $(0,2007)$ \\
$5 \pi / 6(1)$ & LPFR9t & $-0,9652$ & $(0,0033)$ \\
$5 \pi / 6(2)$ & LPFR10t & $-0,7811$ & $(0,1780)$ \\
Anual (1) & LPFR11t & $-0,9659$ & $(0,0032)$ \\
Anual (2) & LPFR12t & $-0,3849$ & $(0,0770)$ \\
\hline
\end{tabular}

Fuente: elaboración propia.

La elasticidad de transmisión en el largo plazo es de $0,9671 \%$; por tanto, se espera que ante un cambio de un $1 \%$ en los precios de futuros de ganado bovino en pie, los precios en Chile cambien, en promedio, en esa proporción. Esta elasticidad es similar a la obtenida por Arias y Segura (2001), que en maíz obtuvieron un coeficiente de $0,785 \%$ y en trigo $0,957 \%$ respecto del mismo mercado futuro.

Una vez determinada la existencia de cointegración estacional, se estimó el modelo de corrección de errores con la metodología Johansen; la Tabla 11 presenta los resultados de la estimación junto con los principales estadísticos de significancia y bondad de ajuste del modelo.

Antes de iniciar el análisis, hay tres resultados interesantes que distinguen al modelo de la estimación obtenida por el método de Engle y Granger (1987). Primero, el modelo estimado es estable pese a la variabilidad de ambas series de precios. El Gráfico 7 presenta las pruebas CUSUM (cumulative sum, por sus siglas en inglés) para el modelo de corrección de errores, en donde se aprecia que no hay signos de inestabilidad en los coeficientes. 
Troncoso y Cabas: Factibilidad del uso de contratos de futuros del Chicago Mercantile...

Tabla 11. Modelo de corrección de errores

\begin{tabular}{lcccc}
\hline \multicolumn{5}{c}{${\text { Variable dependiente: } \text { LPDRS }^{\text {a }}}$} \\
\hline Variable & Coeficiente & Error estándar & Estadístico t & Probabilidad \\
\hline LPFRS & 0,5927 & 0,0312 & 19,0077 & 0,0000 \\
LPDRS(-1) & $-0,6005$ & 0,0368 & $-16,3318$ & 0,0000 \\
LPFRS(-1) & 0,4711 & 0,0311 & 15,1362 & 0,0000 \\
U1t(-1) & 0,0356 & 0,0053 & 6,7745 & 0,0000 \\
U3t(-1) & $-0,0930$ & 0,0217 & $-4,2898$ & 0,0000 \\
U4t(-1) & $-0,3020$ & 0,0287 & $-10,5101$ & 0,0000 \\
U5t(-1) & $-0,2999$ & 0,0308 & $-9,7323$ & 0,0000 \\
U6t(-1) & 0,2692 & 0,0320 & 8,4234 & 0,0000 \\
U8t(-1) & $-0,1145$ & 0,0130 & $-8,7912$ & 0,0000 \\
U9t(-1) & $-0,4112$ & 0,0381 & $-10,8022$ & 0,0000 \\
U10t(-1) & 0,1967 & 0,0450 & 4,3711 & 0,0000 \\
U11t(-1) & 0,0627 & 0,0126 & 4,9612 & 0,0000 \\
U12t(-1) & $-0,0441$ & 0,0064 & $-6,9268$ & 0,0000 \\
DUM & $-0,0254$ & 0,0089 & $-2,8574$ & 0,0045 \\
C & 0,0016 & 0,0034 & 0,4514 & 0,6519 \\
\hline R & 0,6754 & & Estadístico F & 59,8844 \\
R ${ }^{2}$ ajustado & 0,6641 & & Probabilidad & 0,0000 \\
D-W & 1,9946 & & & \\
\hline
\end{tabular}

Nota: ${ }^{a}$ variables diferenciadas.

Fuente: elaboración propia.

Segundo, los residuos del modelo son bien comportados, lo que se puede visualizar a través de pruebas de heteroscedasticidad, autocorrelación y normalidad, como las que se exponen en la Tabla 12.

Las pruebas de heterocedasticidad BPG y ARCH no permiten rechazar la hipótesis nula, por lo que denotan un comportamiento homocedástico e independiente de la varianza respecto de sus rezagos. El estadístico de la prueba de autocorrelación serial LM muestra que no existe correlación residual de orden 12. Finalmente, la prueba Jarque-Bera indica que los residuos del modelo siguen una distribución aproximadamente normal. 
Gráfico 7. Pruebas CUSUM y CUSUM cuadrado
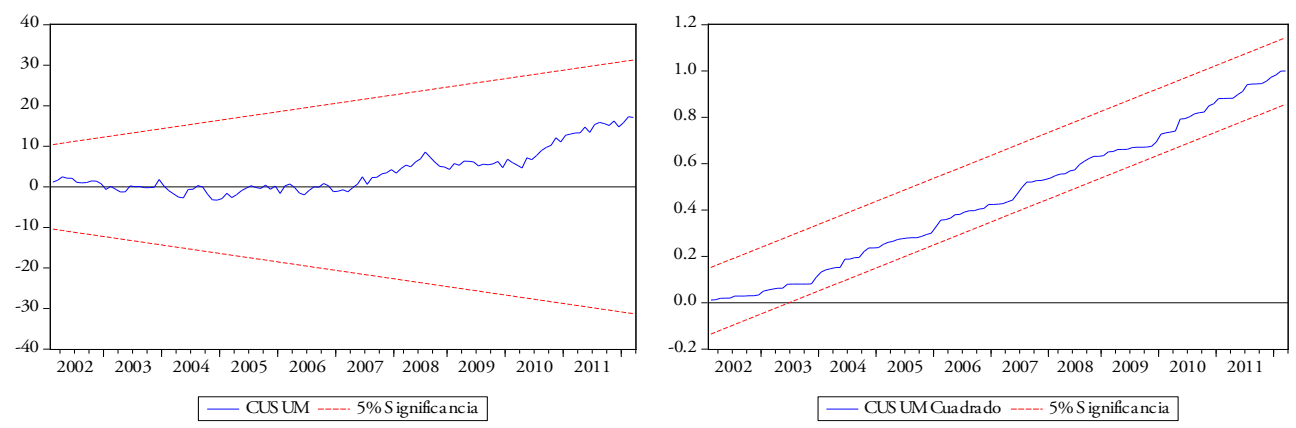

Fuente: elaboración propia.

Tabla 12. Análisis de residuos del modelo de corrección de errores Johansen

\begin{tabular}{lcl}
\hline \multicolumn{2}{l}{ Prueba de heterocedasticidad Breusch-Pagan-Godfrey (BPG) } \\
\hline Estadístico F & 1,8798 & Prob. F(14.403) \\
Obs*R-cuadrado & 25,6229 & Prob. Chi-cuadrado(14) \\
Scaled explained SS & 22,3647 & Prob. Chi-cuadrado(14) \\
\hline \multicolumn{2}{l}{ Prueba de heterocedasticidad ARCH } & \\
\hline Estadístico F & Prob. F(1.405) \\
Obs*R-cuadrado & 1,7507 & Prob. Chi-cuadrado(1) \\
\hline \multicolumn{2}{l}{ Prueba de autocorrelación serial Breusch-Godfrey (LM) } & \\
\hline Obs*R-cuadrado & Prob. Chi-cuadrado(12) \\
\hline Prueba de normalidad Jarque-Bera & \\
Estadístico JB & 3,0000 & Prob. JB \\
\hline
\end{tabular}

Fuente: elaboración propia.

Tercero, el modelo de corrección de errores se encuentra correctamente especificado según los resultados de la prueba Reset de Ramsey (Tabla 13). Esto significa que el modelo se ajusta correctamente al comportamiento de las variables y que todas las que incluye son relevantes. 
Troncoso y Cabas: Factibilidad del uso de contratos de futuros del Chicago Mercantile...

Tabla 13. Prueba reset de Ramsey

\begin{tabular}{lcc}
\hline & Valor & DF \\
\hline Estadístico $t$ & 0,5097 & 402 \\
Estadístico F & 0,2598 & $(1.402)$ \\
Razón de Verosimilud & 0,2701 & 1 \\
\hline
\end{tabular}

Fuente: elaboración propia.

El análisis de los resultados revela que la bondad de ajuste del modelo es relativamente alta $(0,6754)$, que la prueba $F$ acepta la hipótesis de significancia global y que las pruebas $t$ indican que los coeficientes asociados a las variables y a los términos de corrección de error son estadísticamente significativos al $1 \%$.

La elasticidad de corto plazo de los precios de disponible (ajustados estacionariamente) con respecto a los precios de futuro es inelástica y de menor magnitud que la de largo plazo. Un aumento de $1 \%$ en los precios futuros genera, en promedio, un aumento de los precios disponibles de $0,5927 \%$ en el mismo período y de $0,4711 \%$ después de un mes. Desde un punto de vista económico-financiero, esto es consistente con la relación de precios esperada y corrobora que ambos mercados están integrados.

El grado de integración entre los dos mercados también se puede observar analizando la velocidad de convergencia en cada frecuencia estacional a través de los términos de corrección de error $\left(U_{i t}\right)$. En el largo plazo $\left(U_{1 t}\right)$, el coeficiente $\gamma_{1}$ es positivo y altamente significativo, lo que indica que los precios de disponible se encontraban por debajo de su valor de equilibrio en el período $t-1$ y se ajustaron $0,0356 \%$ un mes después de haberse presentado el shock exógeno (Tabla 11). El segundo coeficiente de ajuste cuatrimestral $\left(U_{4 t}\right)$ muestra una velocidad de convergencia hacia el equilibrio de $-0,3020 \%$; esto indica que, en el cuatrimestre anterior, los precios de disponible se encontraban por encima del equilibrio y se ajustaron a dicha tasa un cuatrimestre después. La convergencia más rápida ocurre en el primer coeficiente de la frecuencia $5 \pi / 6$ y es de $-0,4112 \%$; su interpretación es análoga a los casos anteriores. 


\section{F. Cobertura óptima}

A partir de los resultados del modelo de corrección de errores, se estimó la cobertura óptima de mínimo riesgo con contratos futuros para un productor de ganado bovino en un $23,78 \% \%^{6}$. Esta tasa está en línea con el coeficiente de correlación entre los dos mercados $(0,3627)$ y con los óptimos que obtuvieron Arias y Segura (2001) en maíz (24,86 \%) y trigo (30,07\%).

Para corroborar que el porcentaje de cobertura permite reducir el riesgo de precio, se realizó un ejercicio de simulación similar al practicado por Arias y Segura para coberturas de trigo chileno en la Bolsa de Buenos Aires, durante el período 1994:1-2000:8. El supuesto es que un productor ganadero vende una unidad animal ${ }^{7}(450 \mathrm{~kg})$ en el mercado de futuros, y un mes después cierra la operación, lo que le genera una ganancia o una pérdida neta que se suma o se resta al ingreso obtenido por la venta de dicha cantidad en el mercado de contado. Con estas simulaciones, se obtuvieron los flujos mensuales de ingreso que un productor hubiera obtenido en el período 1975:1-2012:3, para distintos niveles de cobertura. En la Tabla 14 se muestra un resumen de los resultados del ejercicio de simulación.

Claramente, se observa que un nivel de cobertura mayor reporta ingresos, en promedio, menores. La desviación estándar o variabilidad de los ingresos es mínima al nivel de cobertura encontrado en el Modelo de Corrección de Errores (23,78\%), lo cual no implica que no existan niveles de cobertura mayores o menores en algunos meses, sino que, simplemente, representa un promedio que permite estabilizar al máximo los precios recibidos por un productor.

En el Gráfico 8 se muestran los resultados reportados en la Tabla 14. En el Cuadrante I se aprecia, inicialmente, que al aumentar el porcentaje de cobertura (desde $0 \%$ ), la desviación estándar de los ingresos disminuye en forma decreciente hasta alcanzar el valor mínimo de US $\$ 146,01$ y, a partir de ahí, todo aumento en el porcentaje de cobertura implica un incremento de

6 La cobertura se obtuvo a partir de coeficiente de elasticidad de largo plazo (frecuencia cero).

7 Para facilitar el análisis, se realizó la simulación en unidades animales (UA). Un contrato de futuros en el Chicago Mercantile Exchange equivale a $40.000 \mathrm{lb}$, aproximadamente 18 toneladas. 
Troncoso y Cabas: Factibilidad del uso de contratos de futuros del Chicago Mercantile...

Tabla 14. Cobertura óptima de minima varianza. Ejercicio de simulación

\begin{tabular}{ccc}
\hline Porcentaje de cobertura & Ingreso promedio & Desviación estándar \\
\hline 100 & 457,86 & 149,55 \\
95 & 457,95 & 149,15 \\
90 & 458,05 & 148,77 \\
85 & 458,14 & 148,42 \\
80 & 458,24 & 148,09 \\
75 & 458,33 & 147,78 \\
70 & 458,42 & 147,50 \\
65 & 458,52 & 147,24 \\
60 & 458,61 & 147,00 \\
55 & 458,71 & 146,79 \\
50 & 458,80 & 146,60 \\
45 & 458,90 & 146,44 \\
40 & 458,99 & 146,30 \\
35 & 459,09 & 146,19 \\
30 & 459,18 & 146,09 \\
23,78 & 459,30 & 146,01 \\
20 & 459,41 & 146,04 \\
15 & 459,52 & 146,06 \\
10 & 459,64 & 146,07 \\
5 & 459,76 & 146,16 \\
0 & 459,84 & 146,23 \\
\hline
\end{tabular}

Fuente: elaboración propia con base en Arias y Segura (2000).

la desviación estándar a una tasa creciente. El Cuadrante II muestra la relación inversa entre el porcentaje de cobertura y el ingreso promedio esperado. Niveles de cobertura mayores al óptimo encontrado implican ingresos, en promedio, menores y tasas de riesgo mayor; mientras que, si bien los niveles de cobertura menores reportan ingresos más altos, estos son a costa de riesgo mayor. La línea de $45^{\circ}$ en el Cuadrante III permite trasladar el ingreso desde el eje vertical hacia el eje horizontal para obtener, en el Cuadrante IV, su relación con la desviación estándar. Este último cuadrante muestra que ingresos 
mayores o inferiores a los US $\$ 459,3$ llevan aparejadas desviaciones estándar mayores, sin estar asociados a coberturas óptimas.

Gráfico 8. Simulación de cobertura. Ingreso y desviación estándar, 1975:1-2012:3

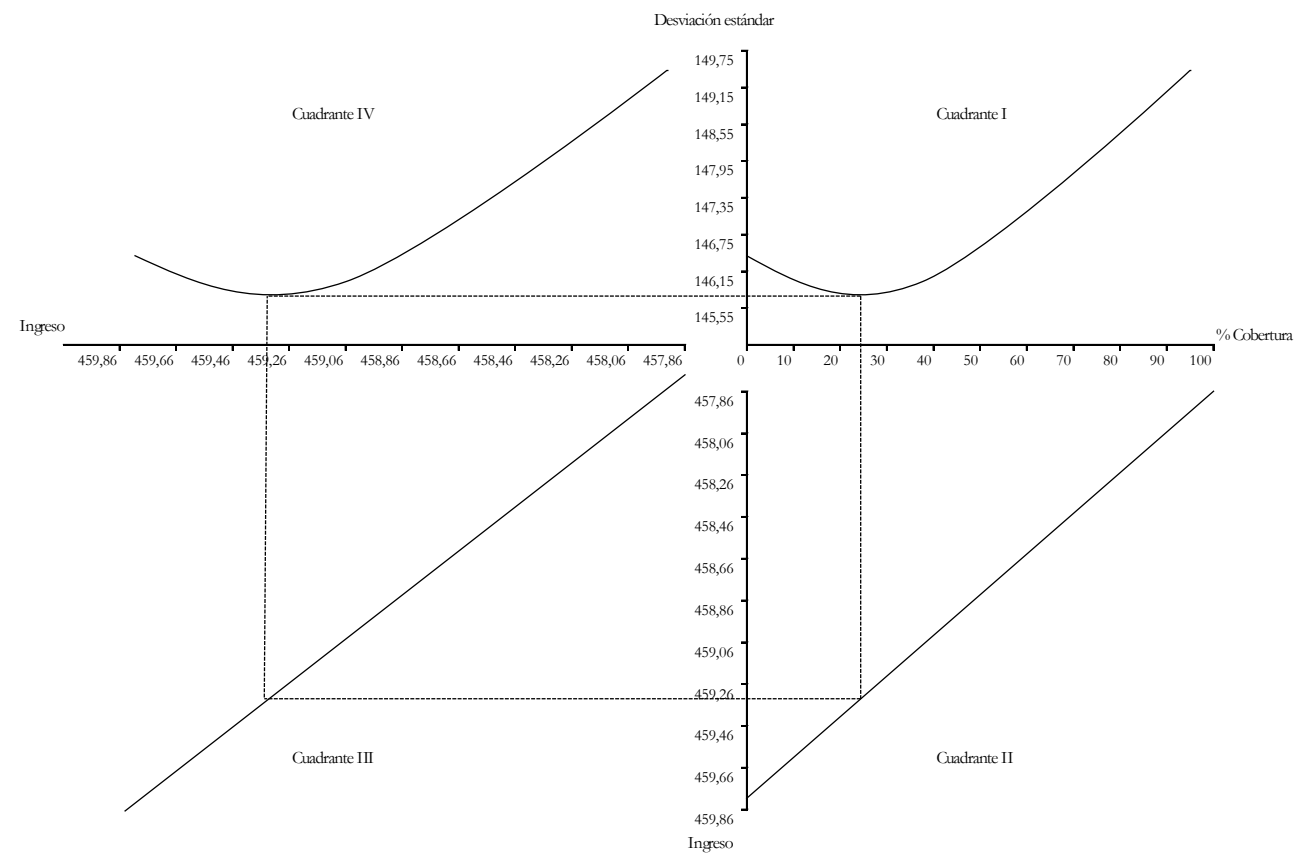

Fuente: elaboración propia con base en Arias y Segura (2000).

El Gráfico 8 ilustra claramente que la cobertura óptima reportaría el mínimo riesgo de ingreso a un productor que decidiera cubrirse en el mercado de futuros. Se podría decir que un productor que realizó coberturas en el período 1975:1-2012:3 obtuvo, en un 23,78\% de las veces, variaciones de los precios futuros versus precios de disponible que le fueron favorables.

El porcentaje de cobertura óptima (e histórica) y la magnitud del contrato de futuro para ganado vivo en el Chicago Mercantile Exchange imponen inmediatamente restricciones sobre el tamaño de la masa ganadera de los potenciales cobertores. Un contrato de futuros es equivalente a 40.000 libras o 18.143,69 kilos (40,32 UA aproximadamente), por tanto, solo productores con masas ganaderas iguales o superiores a las 168.208,93 libras o los 
Troncoso y Cabas: Factibilidad del uso de contratos de futuros del Chicago Mercantile...

76.298,12 kilos (169,55 UA aproximadamente) podrían cubrir con contratos futuros el porcentaje óptimo calculado.

\section{Conclusiones}

El propósito de este trabajo fue determinar la factibilidad de uso de futuros financieros para la cobertura de riesgo de volatilidad de precio en el mercado bovino chileno. Para ello, se estudió la integración de los mercados chileno y norteamericano a través de un análisis de cointegración estacional y se determinó la tasa de cobertura óptima según el modelo de Johnson-Stein.

El mercado bovino nacional y el Chicago Mercantile Exchange se encuentran integrados estacionalmente en las frecuencias cero (largo plazo), cuatrimestral, trimestral, semestral, $5 \pi / 6$ y anual. Existe una relación de causalidad unidireccional y son los precios futuros los que causan a los de disponible, y no a la inversa. Se estimó la elasticidad de largo plazo en $0,9671 \%$; por tanto, ante un cambio del $1 \%$ en los precios de futuros, se esperaría que los precios en Chile cambien, en promedio, en esa proporción. La elasticidad en frecuencia semestral (primer coeficiente de ajuste) es la única que presentó un comportamiento elástico, con un coeficiente de 1,0594\%.

Se descubre que la elasticidad de corto plazo entre los dos mercados es inelástica y considerablemente menor a las de largo plazo y frecuencias estacionales. Un aumento del $1 \%$ en los precios futuros generaría un aumento contemporáneo en los precios de disponible de $0,5927 \%$ y de $0,4711 \%$, transcurrido un mes.

Las elasticidades de transmisión de precios mostraron que en la frecuencia cero los precios de disponible se encuentran, en promedio, por debajo de su valor de equilibrio en el período anterior y se ajustan $0,0356 \%$ un mes después de haberse presentado un shock externo.

El nivel de cobertura óptimo hallado, a partir del ajuste del modelo JohnsonStein, es del $23,78 \%$ de su masa ganadera total y permite asegurar un riesgo mínimo por volatilidad de precios.

A la luz de los resultados, se puede concluir que es técnicamente factible el uso de futuros en productores bovinos nacionales porque los mercados 
están integrados y existe una relación de causalidad, así como una cobertura óptima capaz de reducir el riesgo por volatilidad de precios. Las mayores dificultades siguen siendo el escaso conocimiento de estos instrumentos por parte de los productores, el bajo fomento de las autoridades y organismos, el número escaso de planteles con masas ganaderas que les permitan asegurar, al menos, un contrato y el bajo nivel de asociatividad en los productores.

Se recomienda, en estudios posteriores, utilizar modelos de cobertura que se enfoquen en la maximización de beneficios, y que incorporen factores como la aversión al riesgo, los costos de transacción con futuros (apertura-cierre y comisión corredora), márgenes o garantías y riesgos de base y tipo de cambio. También es recomendable replicar la investigación en otros productos como cerdos magros, leche, maíz, trigo y soya (harina y aceite).

\section{Referencias}

Arias, Joaquín \& Segura, Oswaldo (2000). Factibilidad de uso de los Mercados de Futuros como Instrumento de Cobertura en Bolsas regionales de América Latina: El Caso de la Bolsa de Cereales de Buenos Aires. Buenos Aires: Instituto Interamericano de Cooperación Agrícola.

Cecchetti, Stephen R.; Cumby, Robert \& Figlewski, Stephen (1988). "Estimation of the optimal futures hedge", Review of Economics and Statistics, Vol. 70, No. 4, pp. 623-630.

Cheng-Few, Lee; Kehluh, Wang \& Yang-Long, Chen (2009). "Hedging and Optimal Hedge Ratios for International Index Futures Markets", Review of Pacific Basin Financial Markets and Policies, Vol. 12, No. 4, pp. 593-610.

Cochrane, John (1991). "A critique of the application of unit root tests", Journal of Economic Dynamics and Control, Vol. 15, No. 2, pp. 275-284.

Conlon, Thomas; Cotter, John \& GençAy, Ramazan (2016). "Commodity futures hedging, risk aversion and the hedging horizon", The European Journal of Finance, Vol. 22, No. 15, pp. 1534-1560. 
Troncoso y Cabas: Factibilidad del uso de contratos de futuros del Chicago Mercantile...

Da Silva, Andréia; Aguiar, Danilo \& De Lima, João Eustáquio (2003). "Hedging with futures contracts in the brazilian soybean complex: BM\&F vs. CBOT", Revista de Economía e Sociología Rural, Vol. 41, No. 2, pp. 383-405.

Dickey, D.; Hasza, D. \& Fuller, W. (1984). “Testing for unit roots in seasonal time series", Journal of the American Statistical Association, Vol. 79, No. 386, pp. 355-367.

DICTUC (2011). Evaluación de estrategias alternativas de cobertura ante fluctuaciones de precios de productos agricolas: Estudio del mercado del trigo en Chile y en el extranjero. Santiago: Pontificia Universidad Católica de Chile.

EDERINGTON, Louis (1979). "The hedging performance of the new futures markets", Journal of Finance, Vol. 34, No. 1, pp. 157-170.

Engle, Robert \& Granger, Clive (1987). "Co-Integration and Error Correction: Representation, Estimation, and Testing", Econometrica, Vol. 55, No. 2, pp. 251-276.

Gallardo, Mauricio \& Rubio, Hernán (2009). “Diagnóstico de Estacionalidad con X-12-ARIMA”, Estudios Económicos Estadísticos, No. 76. Banco Central de Chile.

Granger, Clive (1980). "Some properties of time series data and their use in econometric model specification", Journal of Econometrics, Vol. 16, No. 1, pp. 121-130.

Granger, Clive \& Newbold, Paul (1974). "Spurious regressions in econometrics", Journal of Econometrics, Vol. 2, No. 2, pp. 111-120.

Howard, Charles \& D’Antonio, Louis (1984). “A risk-return measure of hedging effectiveness", Journal of Financial and Quantitative Analysis, Vol. 19, No. 1, pp. 101-112.

Hsin, Chin-Wen; Kuo, Jerry \& Lee, Cheng-Few (1994). "A new measure to compare the hedging effectiveness of foreign currency futures versus options", Journal of Futures Markets, Vol. 14, No. 6, pp. 685-707. 
Hylleberg, Svend; Engle, Robert; Granger, Clive \& Yoo, Byung Sam (1990). "Seasonal Integration and Co-Integration", Journal of Econometrics, Vol. 44, No. 1-2, pp. 215-238.

Johansen, Søren \& Juselius, Katrina (1990). "Maximum likelihood estimation and inference on cointegration - with applications to the demand for money", Oxford Bulletin Of Economics and Statistics, Vol. 52, No. 2, pp. 169-210.

Johnson, Leland (1960). "The Theory of Hedging and Speculation in Commodity Futures", Review of Economic Studies, Vol. 27, No. 3, pp. 139151.

MacKinnon, James (1991). "Critical values for cointegration tests”. En: Engle, R. \& Granger, C. Long-run economic relationships: Readings in cointegration (pp. 267-276). Oxford; New York: Oxford University Press.

Manzana, Vicent Aragó (2009). “Teorías sobre cobertura con contratos de futuro", Cuardernos de Economía, Vol. 28, No. 50, pp. 157-190.

Mas, María Sol (2001). "Regulaciones en los mercados de futuros", Investigación \& Desarrollo. Departamento de Capacitación y Desarrollo de Mercados, Bolsa de Comercio De Rosario, Argentina. Recuperado de: http://www.bcr.com.ar/Publicaciones/investigaciones/regulaciones_mas. pdf (agosto de 2013).

Milanesi, Gastón; Miliozzi, Claudio \& Esandi, Juan (septiembre, 2010). "El ratio de cobertura y el riesgo de precio, Aplicaciones en futuros de trigo". Sociedad argentina de docentes. XXX Jornadas Nacionales de Adminstrción Financiera. Córdoba, Argentina.

Myers, Robert \& Thompson, Stanley (1989). “Generalized Optimal Hedge Ratio Estimatio", Michigan Agricultural Experiment Sation Journal, Vol. 71, No. 2, pp. 858-868.

Noussinov, Mikhail \& Leuthold, Raymond (1999). "Optimal Hedging Strategies for the U.S. Cattle Feeder”, Journal of Agribusiness, Vol. 17, No. 1, pp. 1-19. 
Troncoso y Cabas: Factibilidad del uso de contratos de futuros del Chicago Mercantile...

Peña, Daniel (2010). Análisis de Series Temporales. Madrid: Alianza Editorial.

Rolfo, Jaques (1980). "Optimal Hedging under Price and Quantity Uncertainty: The Case of a Cocoa Producer", Journal of Political Economy, Vol. 88, No. $1,100-116$.

Soto, Raimundo \& TAPiA, Matías (2000). "Cointegración Estacional en la Demanda de Dinero", Economia Chilena, Vol. 3, No. 3, pp. 57-71.

Stein, Jerome (1961). "The Simultaneous Determination of Spot and Futures Prices", The American Economic Review, Vol. 51, No. 5, pp. 1012-1025.

Wolf, Avner (1987). "Optimal Hedging with Futures Options", Journal of Economics and Business, Vol. 39, No. 2, pp. 141-158.

Working, Holbrook (1953). "Futures traiding and hedging", American Economic Review, Vol. 43, No. 3, pp. 314-343.

Zurita, Salvador (2002). "Sobre la cobertura de mínima varianza con futuros", Estudios de Administración, Vol. 9, No. E2, pp. 1-14. 